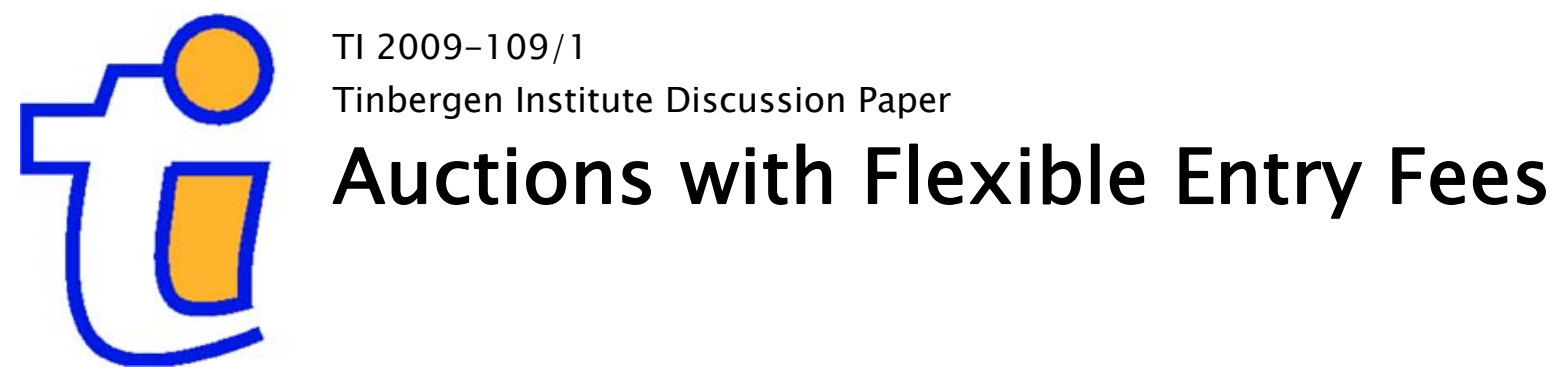

Maarten C.W. Janssen ${ }^{1,2,3}$
Vladimir A. Karamychevi,2

Emiel Maasland ${ }^{1}$

' Erasmus School of Economics, Erasmus University Rotterdam;

2 Tinbergen Institute;

3 University of Vienna. 


\section{Tinbergen Institute}

The Tinbergen Institute is the institute for economic research of the Erasmus Universiteit Rotterdam, Universiteit van Amsterdam, and Vrije Universiteit Amsterdam.

Tinbergen Institute Amsterdam

Roetersstraat 31

1018 WB Amsterdam

The Netherlands

Tel.: +31(0)205513500

Fax: $+31(0) 205513555$

Tinbergen Institute Rotterdam

Burg. Oudlaan 50

3062 PA Rotterdam

The Netherlands

Tel.: + $31(0) 104088900$

Fax: $+31(0) 104089031$

Most TI discussion papers can be downloaded at http://www.tinbergen.nl. 


\title{
Auctions with Flexible Entry Fees ${ }^{1}$
}

\author{
Maarten C.W. Janssen ${ }^{\dagger}$, Vladimir A. Karamychev ${ }^{\ddagger}$, Emiel Maasland ${ }^{\S}$
}

November 2009

\begin{abstract}
There is by now a large literature arguing that auctions with a variety of after-market interactions may not yield an efficient allocation of the objects for sale, especially when the bidders impose strong negative externalities upon each other. This paper argues that these inefficiencies can be avoided by asking bidders prior to the auction to submit any public payment they would like to make. These payments, so-called flexible entry fees, do not affect the allocation decision of the auctioneer. We show that auctions with flexible entry fees have a fully revealing equilibrium where bidders signal their type before the auction itself takes place.
\end{abstract}

JEL classification: C72, D44.

Keywords: auctions, efficiency, entry fee, negative externality, revenue.

$\dagger$ Department of Economics, University of Vienna, and Tinbergen Institute Rotterdam, Hohenstaufengasse 9, 1010 Vienna, Austria, email: maarten.janssen@univie.ac.at.

* Department of Economics and Tinbergen Institute, Erasmus University Rotterdam, Burg. Oudlaan 50,3062 PA Rotterdam, The Netherlands, email: karamychev@ese.eur.nl.

$\S$ SEOR and Department of Economics, Erasmus University Rotterdam, Burg. Oudlaan 50, 3062 PA Rotterdam, The Netherlands, email: emaasland@ese.eur.nl.

1 The authors thank seminar participants at Erasmus University Rotterdam, Martin Perry, and Benny Moldovanu for helpful comments on an earlier version of this paper. 


\section{Introduction}

A relatively recent literature has studied how post-auction interactions, such as resale or after-market competition, affect bidders' bidding behavior in auctions. One application that has received particular attention, both in the theoretical literature and in the popular press, are the auctions for third generation mobile telecommunication licenses around the world, which enable the winning bidders to compete with each other by offering telecommunication services to final consumers. ${ }^{2}$ There are now many papers studying this framework, and most of these papers emphasize that common auction properties do not hold in such an environment and that inefficient outcomes are likely to result.

The literature the present paper builds on studies auctions with the presence of negative informational externalities due to after-market interactions (see Jehiel and Moldovanu, 2006, for an overview). One application of this literature (see Goeree, 2003, Das Varma, 2003, and Moldovanu and Sela, 2003) is a single-unit auction where one object, namely a patent for a cost reduction, is auctioned and the winner competes in the market after the auction with all non-winners. Moldovanu and Sela (2003) show that standard auctions lead to inefficient allocations when bidders' values are strongly and negatively interdependent. ${ }^{3}$ The reason why efficient equilibria do not exist is that on the margin, due to the strong negative externality, the willingness to pay of a more efficient firm is lower than that of a less efficient firm.

Goeree (2003) and Das Varma (2003) analyze a similar setting but allow for signaling private information through the auction bid. The reason why an efficient equilibrium may not exist in these papers is that under strategic complementarity, a more efficient firm may want to understate its private information by shading its bid in order to relax after-market competition. This phenomenon has been documented as a "fat cat" business strategy in Fudenberg and Tirole (1984). Das Varma (2003) shows that the inefficiency gradually disappears when the downstream market becomes perfectly competitive. Katzman and Rhodes-Kropf (2008) analyze how different bidannouncement policies affect the efficiency and revenue of an auction (see also Molnar

\footnotetext{
2 For easily accessible theoretical articles on the main issues involved, see Binmore and Klemperer (2002), Börgers and Dustmann (2003), Klemperer (2002a), Klemperer (2002b), and Van Damme (2002). For a popular press article, see Klemperer (2000).

3 See also Jehiel et al. (1996) and Jehiel and Moldovanu (2000) for related papers where an (informational) externality may lead to inefficiency in standard single-unit auctions.
} 
and Virag, 2008). In particular, Katzman and Rhodes-Kropf (2008) show that when signaling reduces the revenue and threatens the efficiency due to, e.g., strategic complementarity, auctioneers prefer auction formats that do not reveal the winning bid.

Another instance of this literature studies auctioning of multiple objects. Hoppe et al. (2006) concentrate on auctions where bidders are ex-ante asymmetric such as in markets with incumbents and entrants. The main insight in Hoppe et al. (2006) is that auctioning more licenses does not necessarily induce a higher degree of competitiveness, i.e., higher after-market efficiency. Janssen and Karamychev (2009a) show that a negative externality (and associated with it potential allocative inefficiency) may appear when firms differ in their attitudes toward risk. Janssen and Karamychev (2009b) show that when bidders' types are ex-ante correlated, efficient equilibria may fail to exist even when the negative externality is weak. The main reason for this is that the correlation and the externality are, to a certain degree, alternative ways to create conditions for the nonexistence of monotone equilibria.

All these papers differ in many details, such as whether one or multiple objects are auctioned, whether bidders are ex-ante symmetric or asymmetric, whether market demand is certain or uncertain, whether risk attitude plays a role or does not, which auction format is used, etc. In all these environments, efficient equilibria may fail to exist. In the present paper, we study a general model that encompasses many of the environments studied in the literature. We show that in all such environments an auction exists that possesses an efficient equilibrium. In this particular auction, bidders are asked to pay any publicly observable sum of money they would like. We call these voluntary payments "flexible entry fees".

The idea of a voluntary entry fee could be traced back to Maskin and Riley (1981). The voluntary entry fee in that paper, however, is very different from our flexible entry fee. In Maskin and Riley (1981), the auctioneer sets a fixed (inflexible) entry fee and bidders can decide whether to pay that fee or not. The object is then allocated to the highest bidder who has paid the entry fee, if any, and if no bidder has paid the entry fee the object is allocated to the highest bidder. In our paper, bidders decide themselves on the amount of the entry fee they pay (flexibility), and the only thing the auctioneer does is that he collects and announces the entry fees individual bidders have paid. Independent of the chosen entry fee, all bidders are allowed to participate in the auction.

The flexible entry fee gives bidders a possibility to signal their type. The incentive to reveal their types is exactly the reason why bidders may pay a positive entry fee. 
Signaling types through bidding behavior during an auction is usually detrimental to the efficiency of the auction (see, e.g., Goeree, 2003, and Das Varma, 2003). Signaling prior to the auction, however, turns out to have the opposite effect.

The intuition is as follows. First, note that due to negative interdependencies, firms' values are negatively related to the types of other firms. For example, if firms compete à la Bertrand or Cournot in an after-market and a firm's type is its cost efficiency, a firm's valuation for the license, i.e., its after-market profit, negatively depends on the types of the firm's competitors. This negative interdependency creates an incentive for a firm to signal its high efficiency level in order to scare off its competitors in the pre-auction signaling stage so that they bid lower in the auction. The more efficient the firm is, the larger its incentive to signal, because a more efficient firm wins with a higher probability and, therefore, is willing to spend a larger part of its after-market profit on signaling its type through the entry fee. Together with the fact that the after-market profit of this more efficient firm is higher, this implies that the more efficient firm sets a higher entry fee, and the equilibrium is perfectly separating. As a result, all information that is relevant for taking the negative externality into account is revealed before the auction starts, and this information revelation makes the auction efficient. ${ }^{4}$

In the main body of the paper, we show how this auction works in detail for a second-price sealed-bid auction where bidders' valuations negatively depend on the types of the other bidders. For a second-price sealed-bid auction with independently distributed types, we show that if the negative interdependencies are relatively weak, the auction with flexible entry fees is revenue-equivalent to and yields the same (efficient) allocation as the standard second-price sealed-bid auction. If the negative interdependencies are relatively strong, the auction with flexible entry fees remains efficient whereas the standard second-price sealed-bid auction is known to be inefficient. When types are exante affiliated and the affiliation is not too strong, a similar result holds true, but revenue equivalence fails. It turns out that when both auctions have an efficient equilibrium, the auction with flexible entry fees performs better in terms of revenue. In the concluding section, we discuss whether the argument also holds true in other auction formats, such as a first-price sealed-bid auction, or when interdependencies are positive.

Another interpretation of the auction with flexible entry fees (where the monetary fees are collected by the auctioneer) is that firms burn money or hire expensive auction

4 The importance of costly signaling to restore auction efficiency is also studied, although in a very different context, by Schwarz and Sonin (2005). 
experts to signal their strength. As long as the amount of money burnt (the cost of hiring auction experts) is either visible or made public, then this will have the same effect as flexible entry fees. ${ }^{5}$ Again, contrary to the common idea that exchanging information is bad (as it may lead to collusion) ${ }^{6}$, making this kind of information public will improve the efficiency of the auction. This signaling resolves the uncertainty firms have about each other's signals. In this interpretation, however, the revenue collected by the auctioneer is lower than in the standard second-price sealed-bid auction, because a part of the revenue is either burnt or spent on experts.

Apart from the above-mentioned literature, the paper is also related to the literature on auctions with entry fees. Milgrom and Weber (1982) show that entry fees may lead to problems with the existence of monotonic equilibria, and Landsberger and Tsirelson (2000) show that with entry fees or other participation costs, monotonic equilibria become increasingly unlikely once the number of bidders is large. These sources of inefficiency do not arise here because entry fees are flexible so that bidders can decide on the size of the fee they would like to pay. Perry et al. (2000) analyze a two stage sealedbid auction for a single object where the two highest bidders of the first stage proceed to the second stage and all loosing bids are revealed.

The rest of the paper is organized as follows. In Section 2, we describe the basic model with negative externalities by means of an example. In this example, there is one object to be auctioned, there are two bidders with private information about their types, the types are identically and independently distributed, and the bidders have additively separable linear valuation functions with negative interdependencies. In this basic set-up, we show that when the negative interdependency is strong, the auction with flexible entry fees, contrary to a second-price sealed-bid auction, always has an efficient equilibrium. Moreover, we show that in terms of revenues the two mechanisms are equivalent when both have an efficient equilibrium. In Section 3, we show that these results hold in a general setting with independent types. Section 4 analyzes the case of correlated types and Section 5 concludes with a discussion. The appendix contains all proofs.

5 Klemperer (2002a) observes that in the mid nineties Pacific Telephone paid for full page ads in newspapers and hired one of the most prominent auction theorists to give seminars, signaling that the California license was of utmost importance to them.

${ }^{6}$ See, e.g., Grimm et al. (2003) on the role of information provision in facilitating collusion. 


\section{The Basic Model}

As an example, we consider a standard symmetric single-object second-price sealed-bid auction, from now on termed the SP-auction, where two bidders, denoted by $i, j \in\{1,2\}$, $j \neq i$, have interdependent valuations. Bidder $i$ 's type and his value for the object are denoted by $X_{i}$ and $V_{i}$ respectively. The types of the bidders are identically and independently distributed over the interval $[0,1]$ in accordance with a distribution function $F(x) \equiv \operatorname{Pr}\left(X_{i} \leq x\right)$. The values of the bidders for the object are interdependent and given by the following linear valuation function:

$$
V_{i}=v\left(X_{i}, X_{j}\right)=a X_{i}-b X_{j}+c,
$$

where $a>0$ and $c>b>0$, which ensures that values are positive. It is easily seen that the values are negatively interdependent: a bidder i's own type affects his value positively whereas the type of his competitor has a negative effect on his value. This negative dependence reflects the fact that often in an auction where some after-auction interaction (such as after-market competition) takes place, a bidders' type (such as a measure of his cost efficiency) positively affects his own value but negatively inflicts upon the value of the competitor. Moreover, it is easily seen that auction efficiency requires the bidder with the highest type to win the auction.

We make the usual assumptions of a game with private information, namely that a bidder's type is private information, i.e., bidder $i$ knows the realization $x_{i}$ of $X_{i}$, the other bidder $j$ does not know $x_{i}$, but he knows $v$ and $F$, and all this is common knowledge. Invoking a standard procedure, one can easily verify that

$$
\beta^{S P}(x)=(a-b) x+c
$$

constitutes a unique symmetric equilibrium bidding function of the SP-auction provided that $a>b$. This equilibrium is efficient and ensures that the bidder with the highest value makes the highest bid and wins the object. If, on the other hand, $a \leq b$, the function $\beta^{S P}(x)$ decreases so that the SP-auction does not have a monotone symmetric equilibrium. Therefore, there is a strictly positive probability that in this case the SPauction results in an allocation that is inefficient.

The non-standard feature of the mechanism that we consider is that prior to the auction, each bidder decides on an amount $e_{i} \geq 0$ that he voluntarily pays to the seller 
before participating in the auction. We call this $e_{i}$ a flexible entry fee and it is important that this $e_{i}$ is public information before the auction takes place so that bidders can update their beliefs about their competitor's type before the auction starts. We refer to the second-price sealed-bid auction with flexible entry fees as the FEF-auction.

The timing of the game is as follows. In stage one, after nature assigns types to bidders, both bidders simultaneously submit payments $e_{i} \geq 0$ to the auctioneer. These payments are publicly observed. In stage two, the bidders participate in a second-price sealed-bid auction where they submit bids $\beta_{i} \geq 0$, and the bidder with the highest bid gets the object and pays a price that is equal to the second highest bid, which in the case of $N=2$ is also the lowest bid. In case of a tie, which will not happen in equilibrium with positive probability, an arbitrary tie-breaking rule applies. Importantly, only the bids made during the auction (and not the entry fees paid) determine the allocation of the object.

We use Weak Perfect Bayesian Equilibrium, WPBE hereinafter, as the equilibrium concept.

Definition 1. A symmetric WPBE of the FEF-auction consists of a strategy and beliefs such that:

a) the strategy of bidder $i$ is a pair of functions, $e_{i}=e^{*}\left(x_{i}\right)$ and $\beta_{i}=\beta^{*}\left(x_{i}, e_{i}, e_{j}\right)$, where $e_{i}=e^{*}\left(x_{i}\right)$ is the entry fee chosen, and $\beta^{*}\left(x_{i}, e_{i}, e_{j}\right)$ is the bid when $e_{i}$ and $e_{j}$ are the chosen entry fees;

b) the belief of bidder $i$ is the conditional probability distribution $B^{*}\left(y \mid x_{i}, e_{i}, e_{j}\right) \equiv \operatorname{Pr}\left(X_{j} \leq y \mid X_{i}=x_{i}, e_{i}, e_{j}\right)$ of the competitor's type $X_{j}$, conditional on $x_{i}, e_{i}$, and $e_{j}$

c) strategies are optimal given the strategy of the other bidders and beliefs;

d) beliefs are generated by Bayes' rule on-the-equilibrium path.

It is easy to see that $\beta^{S P}(x)$ is also an equilibrium of the FEF-auction, i.e., if $a>b$, then $e^{*}(x)=0, \beta^{*}\left(x, e_{i}, e_{j}\right)=\beta^{S P}(x)$, and naive beliefs $B^{*}\left(y \mid x, e_{i}, e_{j}\right)=F(y)$ on- and off-theequilibrium path, constitute a symmetric equilibrium of the FEF-auction. The reason is as follows. In such a pooling equilibrium, the entry fees that bidders choose are all zero 
and do not contain information on their types. Consequently, the bids are solely based on a bidder's own type. On the other hand, if the bidders anyway do not adjust their bids depending on which entry fees are paid, then there is no point in paying a positive entry fee. Note, however, that this equilibrium does not exist if $a \leq b$.

In addition to the pooling equilibrium, there is another symmetric WPBE of the FEFauction, which is perfectly separating and which always exists. In this equilibrium, bidders choose positive entry fees in accordance with the following increasing and continuously differentiable function: ${ }^{7}$

$$
e^{F E F}(x)=b \int_{0}^{x} F(z) d z=b(x-E(z \mid z<x)) F(x) .
$$

For convenience, we define its generalized inverse function for non-negative values of $e$ as follows:

$$
h(e)=\max \left\{x: e^{F E F}(x) \leq e\right\} .
$$

In other words, if $e \in\left[0, e^{F E F}(1)\right]$ then $h(e)$ is the type which pays entry fee $e$ : $e^{F E F}(h(e))=e$. If, however, $e>e^{F E F}(1)$ then $h(e)$ is defined by $h(e)=1$. The function $h(e)$ represents bidders' beliefs:

$$
B^{F E F}\left(y \mid x, e_{i}, e_{j}\right)=\left\{\begin{array}{l}
0, \text { if } y<h(e) \\
1, \text { if } y \geq h(e)
\end{array} .\right.
$$

In other words, having observed an entry fee $e_{j}$ of bidder $j$, bidder $i$ believes that bidder $j$ is of type $x_{j}=h\left(e_{j}\right)$ with probability one:

$$
\operatorname{Pr}\left(X_{j}=h(e) \mid e_{j}=e\right)=1 \text {. }
$$

It is easy to see that along the equilibrium path, the belief satisfies Bayes' rule.

In the second stage, bidder $i$ bids his value given his belief:

$$
\beta_{i}=\beta^{F E F}\left(x_{i}, e_{i}, e_{j}\right)=a x_{i}-b h\left(e_{j}\right)+c .
$$

We will now argue that (1), (2), (3), and (4) constitute a WPBE. It is clear that if it is an equilibrium, it is efficient as the highest type bidder submits the highest bid and gets the object. Due to full information revelation in the first stage, beliefs are degenerate in the second stage, and bidding one's own valuation is an optimal action in the second-price

\footnotetext{
${ }^{7}$ In the next Section, we comment on the interpretation of this expression for the entry fee.
} 
sealed-bid auction. Hence, bidders do not have a profitable deviation away from bidding $\beta^{F E F}\left(x_{i}, e_{i}, e_{j}\right)$.

We now concentrate on the optimality of paying the flexible entry fee specified in (1). If a bidder $i$ of type $x$ sets entry fee $e^{F E F}(y)$, as if he were of type $y$, then he wins the object (neglecting ties) if the type of the other bidder $z$ satisfies

$$
\beta^{F E F}\left(x, e^{F E F}(y), e^{F E F}(z)\right)>\beta^{F E F}\left(z, e^{F E F}(z), e^{F E F}(y)\right),
$$

which can be written as $a x-b z+c>a z-b y+c$ or

$$
z<\frac{a x+b y}{a+b} .
$$

If this is the case, bidder $i$ wins the object at auction price

$$
\beta_{j}=\beta^{F E F}\left(z, e^{F E F}(z), e^{F E F}(y)\right)=a z-b y+c,
$$

and has valuation $v(x, z)=\beta_{i}=a x-b z+c$. The expected profit of bidder $i$ is therefore

$$
\pi(x, y) \equiv-e^{F E F}(y)+\int_{0}^{(a x+b y) /(a+b)}((a x-b z+c)-(a z-b y+c)) d F(z),
$$

which can be rewritten as follows, by using (1) and integrating by parts:

$$
\begin{aligned}
\pi(x, y) & =-b \int_{0}^{y} F(z) d z+\int_{0}^{(a x+b y) /(a+b)}((a x-b z+c)-(a z-b y+c)) d F(z) \\
& =-b\left(\int_{0}^{y}(y-z) d F(z)\right)+b \int_{0}^{(a x+b y) /(a+b)}(y-z) d F(z)+a \int_{0}^{(a x+b y) /(a+b)}(x-z) d F(z) \\
& =b \int_{y}^{(a x+b y) /(a+b)}(y-z) d F(z)+a \int_{0}^{(a x+b y) /(a+b)}(x-z) d F(z) .
\end{aligned}
$$

Setting the entry fee equal to $e^{F E F}(x)$ is optimal because

$$
\pi(x, x)-\pi(x, y)=a \int_{x-b(x-y) /(a+b)}^{x}(x-z) d F(z)+b \int_{y}^{y+a(x-y) /(a+b)}(z-y) d F(z)>0
$$

for all $y \neq x$. Thus, there is no profitable deviation from $e^{F E F}(x)$, which is lower than $e^{F E F}(1)$. Also, choosing a fee above $e^{F E F}(1)$ is strictly suboptimal, given the proposed beliefs off-the-equilibrium path. Indeed, setting any entry fee $e_{i}>e^{F E F}(1)$ induces the same belief of bidder $j$ as entry fee $e_{i}=e^{F E F}(1): h\left(e_{i}\right)=1$. Thus, raising the entry fee above $e^{F E F}(1)$ neither affects the bid of bidder $j$ (and thus the price to be paid if bidder $i$ 
wins), nor the winning probability of bidder $i$. It only increases his own expenses and is, therefore, strictly suboptimal. Thus, no bidder has an incentive to deviate from $e^{F E F}(x)$.

In fact, any off-the-equilibrium belief supports the equilibrium strategy $e^{F E F}(x)$ and $\beta^{F E F}\left(x_{i}, e_{i}, e_{j}\right)$. Indeed, with any other off-the-equilibrium path beliefs different from $B^{F E F}$, bidder $j$ puts some positive probability that the deviating bidder $i$ is of type $x<1$, whereas $B^{F E F}$ puts zero probability on this event. As a result, the expected value of bidder $j$ and, therefore, his bid, is strictly higher than with the equilibrium belief $B^{F E F}$, which assigns $x=1$ with probability one. This implies that setting $e_{i}>e^{F E F}(1)$ is even less attractive for bidder $i$ if the belief of bidder $j$ differs from $B^{F E F}$. Therefore, any belief supports the strategy $e^{F E F}(x)$ and $\beta^{F E F}\left(x_{i}, e_{i}, e_{j}\right)$ as a WPBE.

Thus, and this is the main point of the example, the FEF-auction has an efficient equilibrium for all values of the parameters $a, b$ and $c$. Moreover, the FEF-auction is never worse (in terms of efficiency) than the SP-auction and is strictly better for some values of the parameters (in particular when $a \leq b$ and the SP-auction is inefficient).

Interestingly, for the case where $a>b$ and both the SP-auction and the FEF-auction have efficient equilibria, they generate equal revenues. In the SP-auction, the revenue comes solely from bids:

$$
\begin{aligned}
R^{S P} & =E\left(\beta^{S P}\left(\min \left\{X_{1}, X_{2}\right\}\right)\right)=E\left(v(z, z) \mid z=\min \left\{X_{1}, X_{2}\right\}\right) \\
& =2 \int_{0}^{1} \int_{0}^{x}(a z-b z+c) d F(z) d F(x)=2(a-b) \int_{0}^{1} \int_{0}^{x} z d F(z) d F(x)+c .
\end{aligned}
$$

In the FEF-auction, to the contrary, a part $R^{F E F, e}$ of the revenue comes from collecting the entry fees:

$$
R^{F E F, e}=2 E\left(e^{F E F}\left(X_{i}\right)\right)=2 \int_{0}^{1} e^{F E F}(x) d F(x)=2 b \int_{0}^{1} \int_{0}^{x} F(z) d z d F(x),
$$

and integrating in parts yields:

$$
R^{F E F, e}=2 b \int_{0}^{1} \int_{0}^{x} F(z) d z d F(x)=2 \int_{0}^{1} \int_{0}^{x} b(x-z) d F(z) d F(x) .
$$

The remaining part $R^{F E F, \beta}$ of the revenue stems from the bids made:

$$
\begin{aligned}
R^{F E F, \beta} & =E\left(\beta^{F E F}\left(z, e^{F E F}(z), e^{F E F}(x)\right) \mid z=\min \left\{X_{1}, X_{2}\right\}, x=\max \left\{X_{1}, X_{2}\right\}\right) \\
& =2 \int_{0}^{1} \int_{0}^{x} v(z, x) d F(z) d F(x)=2 \int_{0}^{1} \int_{0}^{x}(a z-b x) d F(z) d F(x)+c .
\end{aligned}
$$


It can easily be verified that $R^{F E F, e}+R^{F E F, \beta}=R^{S P}$, so that revenue equivalence holds. The intuition for this revenue equivalence is that both auctions are efficient, the lowest type gets zero expected profit, and types are statistically independent, and, consequently, the expressions for revenues in these two auctions are identical.

The fact that the second highest bidder, i.e., the bidder whose bid is relevant for the auction payment, in the FEF-auction shades his bid relative to the bid he would make in the SP-auction follows from (4). Knowing that his competitor has a higher type (as $\left.h\left(e_{j}\right)>x_{i}\right)$ makes bidder $i$ bidding less $\left(\beta^{F E F}=a x_{i}-b h\left(e_{j}\right)+c\right)$ than he would have bid in the SP-auction $\left(\beta^{S P}=a x_{i}-b x_{i}+c\right)$ where he would have bid an amount as if his competitor were of the same type $x_{i}$.

As all bidders have to pay the entry fee they have proposed, it is clear that the winner of the auction is better off in the FEF-auction and all non-winners are worse off. In other words, from the perspective of a bidder of a given type, the FEF-auction provides higher pay-off in case he wins, and lower pay-off in case he loses, than the SP-auction. At the same time, both auctions yield equal expected surplus to the bidder. This implies that, from the bidders' perspective, the FEF-auction is riskier than the SP-auction and, therefore, with risk-averse bidders, the FEF-auction would raise higher revenue than the SP-auction.

A natural question that arises is why bidders want to pay a positive entry fee. The reason is that, although the entry fee is sunk at the moment of the auction, entry fees signal bidders' types thereby affecting each other's bids in a desirable way: bids get lower when entry fees increase. By raising the entry fee, a bidder reduces the bid of his competitor and, therefore, lowers the price to be paid in case he wins the object.

We conclude that the FEF-auction yields the same outcome in terms of efficiency and revenue as the SP-auction when the latter has an efficient equilibrium, but retains the property of efficiency for parameter values where the SP-auction does not have an efficient equilibrium.

\section{The General Model with Independent Types}

The example in the previous section was special in a number of ways: the valuation function was supposed to be linear in the bidders' types, the analysis was restricted to two bidders and one object, and the types were supposed to be independently distributed. In 
this section, we first relax the first two assumptions and show that they are not essential to the argument. The last part of the section analyzes the effect of allowing bidders' types to be correlated. We will see that in that case the argument can only be extended by allowing weak forms of affiliation.

Consider a standard symmetric multi-unit uniform-price auction where $N \geq 2$ bidders with unit demand, denoted by subscript $i$, compete for $n \geq 1$ homogeneous objects, $n<N$. Bidders' types $X_{i}$ are identically and independently distributed over the interval $[0,1]$ in accordance with a distribution function $F(x)$. The values of the bidders for the objects are interdependent and given by the following valuation function:

$$
V_{i}=v\left(X_{i}, \mathbf{X}_{-i}\right) \text {, }
$$

where $\mathbf{X}_{-i}$ is a collection of types of all bidders other than $i$. We assume that $v\left(X_{i}, \mathbf{X}_{-i}\right)$ is symmetric in all $X_{j} \in \mathbf{X}_{-i}$, differentiable on $[0,1]^{N}$, and $\partial v / \partial X_{i}>0>\partial v / \partial X_{j}$, i.e., there is a negative externality. ${ }^{8}$

In the FEF-auction, bidders simultaneously choose and publicly pay entry fees $e_{i}$ and then simultaneously submit auction bids $\beta_{i}$. The $n$ bidders who have submitted the $n$ highest bids get the objects and pay the auction price, which is equal to the highest non-winning bid. We denote the equilibrium bidding function by $\beta_{i}=\beta^{F E F}\left(x_{i}, e_{i}, \mathbf{e}_{-i}\right)$, where $\mathbf{e}_{-i}$ is a collection of entry fees chosen by all bidders other than $i$, and consider symmetric equilibria where $\beta^{F E F}\left(x_{i}, e_{i}, \mathbf{e}_{-i}\right)$ is symmetric in all $e_{j} \in \mathbf{e}_{-i}$.

Suppose that a bidder's type $X_{i}$ takes value $x_{i}$. We denote the bidder with the $n^{\text {th }}$ highest type amongst all $(N-1)$ bidders except bidder $i$ by $k$ so that his type is $x_{k}$. Excluding bidders $i$ and $k$, we refer to all remaining $(n-1)$ bidders of types $x_{j}>x_{k}$ by subscript $w \in \mathbf{W}$ (they all win the auction and get objects), and we refer to $(N-n-1)$ remaining bidders of types $x_{j}<x_{k}$ by subscript $l \in \mathbf{L}$ (they all lose the auction). If $n=1$ then $\mathbf{W}=\varnothing$, and if $N=n+1$ then $\mathbf{L}=\varnothing$.

We define a function $\hat{v}(x, y, z)$ as the expected value of a bidder $i$ of type $x_{i}=x$ conditional on (i) one of his competitors, bidder $j$, being of type $x_{j}=y$, (ii) $(n-1)$

8 In some settings, it is more realistic to assume that values only depend on the types of the winning bidders, e.g., when auction winners compete with each other in an after-market. The analysis tolerates such a setting quite easily. 
bidders $w \in \mathbf{W}$ being of type $x_{w}>z$, and (iii) $(N-n-1)$ bidders $l \in \mathbf{L}$ being of type $X_{l}<\mathrm{Z}:$

$$
\hat{v}(x, y, z) \equiv E\left(v\left(X_{i}, \mathbf{X}_{-i}\right) \mid X_{i}=x, X_{j}=y, X_{w}>z, X_{l}<z\right),
$$

where the expectation is taken with respect to $(n-1)$ random variables $X_{w}$ and $(N-n-1)$ random variables $X_{l}$.

The main proposition of this paper demonstrates that the FEF-auction always has an efficient, i.e., a perfectly separating, monotone, and symmetric, WPBE.

Proposition 1. There exists an efficient WPBE of the FEF-auction, where bidders choose an entry fee:

$$
e^{F E F}(x)=\int_{0}^{x}(\hat{v}(z, z, z)-\hat{v}(z, x, z)) d G(z),
$$

bid according to

$$
\beta^{F E F}\left(x_{i}, e_{i}, \mathbf{e}_{-i}\right)=v\left(x_{i}, \mathbf{h}\left(\mathbf{e}_{-i}\right)\right),
$$

where $\mathbf{h}\left(\mathbf{e}_{-i}\right)$ consists of $h\left(e_{j}\right)=\max \left\{x: e^{F E F}(x) \leq e_{j}\right\}$, and beliefs are given by

$$
B^{F E F}\left(y \mid x, e_{i}, e_{j}\right)=\left\{\begin{array}{l}
0, \text { if } y<h\left(e_{j}\right) \\
1, \text { if } y \geq h\left(e_{j}\right)
\end{array} .\right.
$$

In equilibrium, higher types pay a higher entry fee in the first stage. In the second stage, all bidders bid their values. Proposition 1 shows that all the properties of the unique monotone symmetric perfectly separating WPBE presented in the example of Section 2 continue to hold for an arbitrary valuation function which exhibits a negative externality, and for an arbitrary number of objects and bidders. As in the example, bidders' beliefs off-the-equilibrium path do not play an important role here as any belief supports the separating Bayesian equilibrium strategies as WPBE.

It is interesting to note the interpretation of the entry fee. Let bidder $i$ have the same type as bidder $k$, i.e., $x_{i}=x_{k}=z$. Then, the valuation of the latter would have been $\hat{v}(z, z, z)$. Hence, by having value $x_{i}=x$, bidder $i$ imposes a negative externality of size $\hat{v}(z, z, z)-\hat{v}(z, x, z)$ on bidder $k$ by reducing his value by that amount. This externality only realizes when bidder $i$ wins and bidder $k$ does not win, i.e., when $z<x$. Therefore, from bidder i's perspective, the entry fee $e^{F E F}(x)$ he pays is the expected externality he 
imposes on the marginal bidder $k$. Thus, flexible entry fees allow bidders to internalize the negative externality they impose on each other so that the externality does not affect the monotonicity property of bidders' bids. Hence, an efficient equilibrium always exists. This is the crucial difference between the entry fee chosen by the auctioneer (including the case of a voluntary entry fee à la Maskin and Riley, 1981) and flexible entry fees chosen by bidders themselves.

In case $\hat{v}(x, x, x)$ is an increasing function of $x$, the FEF-auction also has a pooling equilibrium that coincides with the equilibrium of the SP-auction, where all bidders choose an entry fee of zero and bid their expected valuation in case they are uncertain about their competitors' types, i.e., $e^{*}(x)=0$ and $\beta^{*}\left(x, e_{i}, \mathbf{e}_{-i}\right)=\beta^{S P}(x)=\hat{v}(x, x, x)$, and beliefs are the prior beliefs $B^{*}\left(y \mid x, e_{i}, e_{j}\right)=F(y)$. Contrary to the separating equilibrium, the pooling equilibrium requires specific beliefs off-the-equilibrium path. The revenue generated in the separating equilibrium is equal to the revenue generated in the SPauction as revenue equivalence holds. In case $\hat{v}(x, x, x)$ is not monotonically increasing, the SP-auction does not have an efficient equilibrium and, therefore, its outcome is inefficient with positive probability. These results are summarized in the next proposition.

Proposition 2. The SP-auction has an efficient equilibrium if and only if $\hat{v}(x, x, x)$ is an increasing function of $x$, in which case $\beta^{S P}(x)=\hat{v}(x, x, x)$. If an efficient equilibrium exists, it raises the same expected revenue as the separating equilibrium of the FEFauction.

This Section has considered second-price sealed-bid auctions (and auctions that are strategically equivalent) and one may wonder what the results may be if a first-price sealed-bid auction is considered. It is easy to see that a perfectly revealing equilibrium never exists in a first-price sealed-bid auction with negative externalities. This can best be seen in the two bidder's case. If such an equilibrium had existed, the bidder with the highest type would have bid marginally higher than the other bidder who, in turn, would have bid his true value. This outcome is, however, prone to the following deviation. By setting his entry fee equal to zero, bidder one ensures that the other bidder believes in winning the auction. As a result, bidder two will bid not his own value but the value of the first bidder conditional on his type being zero, which is lower. Hence, by setting his 
entry fee equal to zero, bidder one lowers the bid function of bidder two and wins the object with certainty. Besides, he saves on the entry fee. This makes the deviation profitable, and the corresponding separating equilibrium fails to exist. Thus, the secondprice principle is crucial to obtain the separating result we emphasize in this paper.

\section{Correlated Types}

We now generalize our example of Section 2 to an environment where bidders' types are positively correlated, provided the correlation is not too strong. It is clear that positive correlation of types reinforces the negative externality so that an efficient equilibrium of the SP-auction is even less likely to exist (see also, e.g., Janssen and Karamychev, $2009 \mathrm{~b}$ ). The reason is as follows. A first effect of a bidder's type is that a high type bidder has a higher value than a low type bidder, for the same fixed types of their competitors. This first, direct effect is positive. However, due to positive correlation, a high type bidder expects competitors to be of higher types than a low type bidder expects them to be. This creates a second, indirect effect, on the value, which is negative. When the correlation is strong, the second effect dominates the first one so that the ex-ante expected value of a bidder conditional on winning is not a monotonically increasing function of his type. Consequently, as his bid in the SP-auction is his expected value, a monotone bidding equilibrium fails to exist.

In our model where signaling is allowed, if bidders' types are strongly correlated bidders do not have an incentive to signal their types by paying a (high) entry fee as the other bidders can anyway infer someone's type once they have observed their own type. Therefore, an efficient equilibrium of the FEF-auction only exists if the correlation is not too strong.

To study the effects of correlation we consider for simplicity the two-bidder setting of Section 2. Suppose bidders' types are weakly affiliated and the distribution function of $X_{j}$ conditional on $X_{i}=x$ is $F(z \mid x) \equiv \operatorname{Pr}\left(X_{j} \leq z \mid X_{i}=x\right)$, the density is $f(z \mid x)$, and $F_{x}(z \mid x) \equiv \partial F(z \mid x) / \partial x \leq 0$, i.e., there is affiliation. Let the value function be $v\left(X_{i}, X_{j}\right)=a X_{i}-b X_{j}+c$. We consider situations where

$$
F(y \mid y) \geq F\left(\frac{a x+b y}{a+b} \mid x\right) \text { for } y>x, \text { and } F(y \mid y) \leq F\left(\frac{a x+b y}{a+b} \mid x\right) \text { for } y<x,
$$


for all $x, y \in[0,1]$. When types are independent, condition (5) is always satisfied. When $X_{j}$ and $X_{i}$ are affiliated and $y>x$, the distribution $F(\ldots \mid y)$ stochastically dominates $F(\ldots \mid x)$ so that $F(y \mid y)<F(y \mid x)$. Hence, (5) is only satisfied when the affiliation is weak. On the other hand, for a given distribution with affiliation, (5) is never satisfied for $a=0$, which corresponds to the limiting case of an externality that is extremely strong. Hence, (5) assumes both a relatively weak affiliation of types and a weak externality.

The following proposition shows that condition (5) guarantees the existence of an efficient WPBE of the FEF-auction.

Proposition 3. Consider the case where $N=2$ and the value function is linear and given by $v\left(X_{i}, X_{j}\right)=a X_{i}-b X_{j}+c$. If (5) holds (sufficient condition), then the strategy consisting of an entry fee $e^{F E F}(x)$ and bids $\beta_{i}=\beta^{F E F}\left(x_{i}, e_{i}, e_{j}\right)$ with

$$
e^{F E F}(x)=b \int_{0}^{x} F(z \mid z) d z \text { and } \beta^{F E F}\left(x_{i}, e_{i}, e_{j}\right)=a x_{i}-b h\left(e_{j}\right)+c,
$$

where $h\left(e_{j}\right)=\max \left\{x: e^{F E F}(x) \leq e_{j}\right\}$ and beliefs

$$
B^{F E F}\left(y \mid x, e_{i}, e_{j}\right)=\left\{\begin{array}{l}
0, \text { if } y<h\left(e_{j}\right) \\
1, \text { if } y \geq h\left(e_{j}\right)
\end{array},\right.
$$

constitute an efficient WPBE of the FEF-auction.

This WPBE only exists if (necessary condition)

$$
\frac{a}{a+b} f(x \mid x)+F_{x}(x \mid x) \geq 0 \text {. }
$$

The argument made in the proof of Proposition 3 is similar to the one made in Section 2 and replaces the unconditional distribution function used there by the conditional distribution function and then shows that the argument can be extended by allowing weak forms of affiliation of the type that satisfies (5).

In equilibrium, bidders bid their values in the second stage. In the first stage, they pay entry fees that are increasing in types. Condition (5) guarantees that there is no profitable deviation from $e^{F E F}(x)$, i.e., it is essentially a (global) sufficient second-order condition that ensures that further deviations are even less profitable than smaller deviations. The necessary (local) second-order condition for the FEF-auction to have an 
efficient equilibrium can be obtained from (5) by taking a limit when $x$ and $y$ converge to each other.

In accordance with Proposition 3, the FEF-auction does not have an efficient separating equilibrium when the correlation and the externality are strong. Nevertheless, the FEF-auction can have an efficient pooling equilibrium, which is an efficient equilibrium of the SP-auction if it exists. Thus, the FEF-auction is at least as efficient as the SP-auction, and sometimes it is strictly more efficient.

In the case of affiliation, we may also wonder how revenues under the efficient equilibrium of the SP-auction and the efficient equilibrium of the FEF-auction compare. To make the comparison useful, we have to consider situations where both equilibria exist, and therefore we restrict the analysis to the case where (5) holds and, in addition, $a>b$ so that the SP-auction has an efficient equilibrium. It is straightforward to show that the efficient equilibrium of the SP-auction is given by $\beta^{S P}(x)=v(x, x)=(a-b) x+c$. The next proposition shows that the FEF-auction generates larger revenues than the SPauction.

Proposition 4. Consider the case where $N=2$ and the value function is linear and given by $v\left(X_{i}, X_{j}\right)=a X_{i}-b X_{j}+c, a>b, X_{i}$ and $X_{j}$ are affiliated, and (5) holds. Then, revenue in the FEF-auction is strictly higher than in the SP-auction.

In the FEF-auction, the revenue does not only come from the winning bid and the entry fee paid by the winning bidder, but also from the entry fees paid by all other bidders. Therefore, the Linkage (Revenue Ranking) Principle ( $c f$. Krishna, 2002, p. 103) cannot be applied, and the revenue has to be computed and compared with the revenue in the SPauction directly. It turns out that the FEF-auction better exploits the correlation of bidders' types from the perspective of the auctioneer and results in a higher revenue.

\section{Discussion and Conclusion}

In this paper, we have argued that by allowing bidders to make flexible, publicly observable payments before they enter an auction, the negative externalities, which have played an important role in the recent literature on auctions with post-auction interactions, can be mitigated. The literature has stressed that if the negative externalities are strong enough, auctions may not yield an efficient allocation of the object(s). We 
have argued that asking for a flexible entry fee restores efficiency, and in case of affiliated types brings about a higher revenue. Important to note here is that this argument can be generalized to settings with asymmetric bidders, e.g., to bidders with different valuation functions and different distributions of bidders' types. The reason is that in a separating equilibrium bidders just bid their true values irrespective of whether there are asymmetries between them or not.

We have made the argument in this paper by considering negative externalities. It is easy to see that a perfectly revealing equilibrium never exists in a second-price sealed-bid auction with positive externalities. The reason bidders are willing to pay an entry fee in a setting where externalities are negative is that this has a negative impact on the expected valuation of the other bidders, hence on their bids, and thereby on the price that bidders have to pay for the object in case they win the auction. Under positive externalities, to the contrary, bidders are willing to signal that their types are the lowest possible types as this has a negative impact on the expected valuation of the other bidders, hence on their bids, and thereby on the price that bidders have to pay for the object in case they win the auction. Consequently, if the externality is positive only pooling equilibria exist, in which bidders do not pay an entry fee and then play the standard second-price sealed-bid auction. The type of applications that motivate this paper, however, like auctions with Cournot and Bertrand type of competition in the downstream market are all examples where negative externalities are present.

In summary, we have shown that if the auction itself is efficient, the efficiency of the auction will not be affected by the introduction of flexible entry fees. If the auction is, to the contrary, inefficient, then the introduction of flexible entry fees restores the efficiency of the auction by allowing bidders to signal their type prior to the auction. 


\section{References}

Binmore, K., Klemperer, P., 2002. The biggest auction ever: the sale of the British 3G telecom licenses. Economic Journal 112(478), 74-96.

Börgers, T., Dustmann, C., 2003. Awarding telecom licenses: the recent European experience. Economic Policy 18(36), 215-268.

Das Varma, G., 2003. Bidding for a process innovation under alternative modes of competition. International Journal of Industrial Organization 21(1), 15-37.

Fudenberg, D., Tirole, J., 1984. The fat-cat effect, the puppy-dog ploy, and the lean and hungry look. American Economic Review 74(2), 361-366.

Goeree, J., 2003. Bidding for the future. Journal of Economic Theory 108(2), 345-364.

Grimm, V., Riedel, F., Wolfstetter, E., 2003. Low price equilibrium in multi-unit auctions: the GSM spectrum auction in Germany. International Journal of Industrial Organization 21(10), 1557-1569.

Hoppe, H., Jehiel, Ph., Moldovanu, B., 2006. License auctions and market structure. Journal of Economics and Management Strategy 15(2), 371-396.

Janssen, M.C.W., Karamychev, V., 2009a. Auctions, aftermarket competition, and risk attitudes. International Journal of Industrial Organization 27(2), 274-285.

Janssen, M.C.W., Karamychev, V.A., 2009b. Do auctions select efficient firms? Economic Journal, forthcoming.

Jehiel, Ph., Moldovanu, B., 2000. Auctions with downstream interaction among buyers. Rand Journal of Economics 31(4), 768-791.

Jehiel, Ph., Moldovanu, B., 2006. Allocative and informational externalities in auctions and related mechanisms. In: Blundell, R., Newey, W., Persson, T. (Eds.), The Proceedings of the 9th World Congress of the Econometric Society. Cambridge University Press, Cambridge.

Jehiel, Ph., Moldovanu, B., Stacchetti, E., 1996. How (not) to sell nuclear weapons. American Economic Review 86(4), 814-829. 
Katzman, B., Rhodes-Kropf, M., 2008. The consequences of information revealed in auctions. Applied Economics Research Bulletin (special issue on auctions), Vol. I (March 2008), 53-87.

Klemperer, P., 2000. Sold! The case for auctions, The Wall Street Journal Europe, November 9, p. 11. Available at:

UUhttp://www.nuff.ox.ac.uk/users/klemperer/sold.pdf.

Klemperer, P., 2002a. What really matters in auction design, Journal of Economic Perspectives 16(1), 169-189.

Klemperer, P., 2002b. How (not) to run auctions: the European 3G mobile telecom auctions. European Economic Review 46(4-5), 829-845.

Krishna, V., 2002. Auction theory. San Diego: Academic Press.

Landsberger, M., Tsirelson, B., 2000. Correlated signals against monotone equilibria. SSRN Working Paper. Available at: http://ssrn.com/sol3/abstract=222308.

Maskin, E.S., Riley, J., 1981. The gains to making losers pay in high bid auctions. UCLA WP \# 198. Available at: http://www.econ.ucla.edu/workingpapers/wp198.pdf.

Milgrom, P., Weber, R., 1982. A theory of auctions and competitive bidding. Econometrica 50(5), 1089-1122.

Moldovanu, B., Sela, A., 2003. Patent licensing to Bertrand competitors. International Journal of Industrial Organization 21(1), 1-13.

Molnar, J., Virag, G., 2008. Revenue maximizing auctions with market interaction and signaling. Economics Letters 99(2), 360-363.

Perry, M., E., Wolfstetter and S. Zamir. 2000. A Sealed-Bid Auction That Matches the English Auction, Games and Economic Behavior 33 (2), 265-273

Schwarz, M., Sonin, K., 2005. Efficient actions in a dynamic auction environment, Mimeo, University of California at Berkeley and NBER. Available at: http://rwj.berkeley.edu/schwarz/publications/Schwarz\%20Efficient\%20Actions.pdf.

Van Damme, E. 2002. The European UMTS-auctions. European Economic Review 46(4-5), 846-58. 


\section{Appendix}

\section{Proof of Proposition 1.}

First, it is easy to see that $e^{F E F}(x)$ is a strictly increasing function so that the proposed WPBE is perfectly separating:

$$
\frac{d}{d x} e^{F E F}(x)=-\int_{0}^{x}\left(\frac{d}{d x} \hat{v}(z, x, z)\right) d G(z)=-\int_{0}^{x}\left(\frac{\partial \hat{v}}{\partial y}(z, x, z)\right) d G(z)>0
$$

due to $\partial \hat{v} / \partial y<0$ as being the conditional expectation of $\partial v / \partial X_{j}<0$.

Suppose all bidders except bidder $i$ have beliefs $B^{F E F}$ and follow the proposed WPBE strategy. In this case, each bidder $j$ sets entry fee $e_{j}=e^{F E F}\left(x_{j}\right) \leq e^{F E F}(1)$ and, therefore, bidder $i$ correctly infers the type of all other bidders on-the-equilibrium path by using the inverse function $h\left(e_{j}\right)=x_{j}$. Irrespective of the chosen entry fee $e_{i}$, bidding his exact value $v_{i}=v\left(x_{i}, \mathbf{h}\left(\mathbf{e}_{-i}\right)\right)$ in the second stage is optimal for bidder $i$, just like in the SPauction. Thus, bidder $i$ has no profitable deviation from bidding $\beta^{F E F}\left(x_{i}, e_{i}, \mathbf{e}_{-i}\right)=v\left(x, \mathbf{h}\left(\mathbf{e}_{-i}\right)\right)$. In the rest of the proof, we show that bidders do not benefit by deviating from $e^{F E F}(x)$ for the proposed on- and off-the-equilibrium path beliefs.

For notational convenience, we will write the collection of types other than $X_{i}$ as $\mathbf{X}_{-i}=\left(x_{k}, \mathbf{X}_{-i k}\right)$ referring to the type of a given bidder $k$. By $\hat{F}\left(\mathbf{x}_{-i k} \mid z\right)$ we denote the joint distribution function of $\mathbf{X}_{-i k}$ conditional on $X_{w}>z>X_{l}$ for all other winning bidders $w \in \mathbf{W}$ and losing bidders $l \in \mathbf{L}$. Using this notation, $\hat{v}(x, y, z)$ can be written as follows:

$$
\hat{v}(x, y, z)=\frac{\int_{\substack{X_{w}>z, X_{l}<z \\ X_{i}=x, X_{k}=y}} v\left(X_{i}, \mathbf{X}_{-i}\right) \prod_{\substack{j \neq i \\ j \neq k}} d F\left(x_{j}\right)}{\operatorname{Pr}\left(X_{w}>z, X_{l}<z \mid X_{i}=x, X_{k}=y\right)}=\int_{X_{l}<z<X_{w}} v\left(x,\left(y, \mathbf{X}_{-i k}\right)\right) d^{n-2} \hat{F}\left(\mathbf{x}_{-i k} \mid z\right) .
$$

Suppose bidder $i$ of type $x$ sets entry fee $e_{i}=e^{F E F}(y)$ as if he were of type $y \in[0,1]$, and all other bidders $j$ follow the equilibrium strategy. Bidder $i$ wins and gets the object if and only if his bid $\beta_{i}$ is higher than bid $\beta_{k}$ of bidder $k$. Denoting $x_{k}=z$ and taking into account that $\beta_{i}=v\left(x,\left(z, \mathbf{X}_{-i k}\right)\right)$ and $\beta_{k}=v\left(z,\left(y, \mathbf{X}_{-i k}\right)\right)$, we write $\beta_{i}>\beta_{k}$ as 
$v\left(x,\left(z, \mathbf{X}_{-i k}\right)\right)>v\left(z,\left(y, \mathbf{X}_{-i k}\right)\right)$. If this is indeed the case, bidder $i$ pays the auction price $\beta_{k}$ and gets surplus

$$
s\left(x, y, z, \mathbf{X}_{-i k}\right) \equiv \beta_{i}-\beta_{k}=v\left(x,\left(z, \mathbf{X}_{-i k}\right)\right)-v\left(z,\left(y, \mathbf{X}_{-i k}\right)\right) .
$$

If this is not the case, bidder $i$ does not get the object. The expected surplus $\hat{s}(x, y)$ of bidder $i$ conditional on winning is

$$
\hat{s}(x, y) \equiv E\left(s\left(x, y, z, \mathbf{X}_{-i k}\right) \mid s\left(x, y, z, \mathbf{X}_{-i k}\right)>0\right),
$$

where the expectation is taken with respect to $z$ and $\mathbf{X}_{-i k}$. Bidder i's ex-ante surplus is, therefore,

$$
\pi(x, y) \equiv-e^{F E F}(y)+\operatorname{Pr}\left(s\left(x, y, z, \mathbf{X}_{-i k}\right)>0\right) \cdot \hat{s}(x, y) .
$$

We will show that $\pi_{y}(x, y)<0$ for all $y>x$ and $\pi_{y}(x, y)>0$ for all $y<x$, which implies that $\pi(x, y)$ attains its unique global maximum w.r.t. $y$ at $y=x$.

First, we note that $z<x$ is equivalent to $v\left(z,\left(z, \mathbf{X}_{-i k}\right)\right)>v\left(z,\left(x, \mathbf{X}_{-i k}\right)\right)$ for any realization of $\mathbf{X}_{-i k}$. This is so because the right-hand side is strictly decreasing in $x$ and equals the left-hand side at $x=z$. This allows us to rewrite $e^{F E F}(x)$ as follows:

$$
e^{F E F}(x)=\int_{0}^{x}(\hat{v}(z, z, z)-\hat{v}(z, x, z)) d G(z)=\int_{v\left(z,\left(z: \mathbf{X}_{-i k}\right)\right)>v\left(z,\left(x: \mathbf{X}_{-i k}\right)\right)}(\hat{v}(z, z, z)-\hat{v}(z, x, z)) d G(z) .
$$

Next, we write

$$
\hat{v}(z, z, z)=\int_{X_{l}<z<X_{w}} v\left(x,\left(y, \mathbf{X}_{-i k}\right)\right) d^{n-2} \hat{F}\left(\mathbf{x}_{-i k} \mid z\right),
$$

and

$$
\hat{v}(z, x, z)=\int_{X_{l}<z<X_{w}} v\left(z,\left(x, \mathbf{X}_{-i k}\right)\right) d^{n-2} \hat{F}\left(\mathbf{x}_{-i k} \mid z\right) .
$$

Hence,

$$
\begin{aligned}
e^{F E F}(x) & =\int_{\substack{v\left(z,\left(z, X_{-i k}\right) \\
X_{l}<z<X_{w}\right.}} \iint_{v\left(z,\left(x: X_{-i k}\right)\right)}\left(v\left(z,\left(z, \mathbf{X}_{-i k}\right)\right)-v\left(z,\left(x, \mathbf{X}_{-i k}\right)\right)\right) d^{n-2} \hat{F}\left(\mathbf{x}_{-i k} \mid z\right) d G(z) \\
& =\int_{\substack{s\left(z, x, Z, X_{-i k}>0 \\
X_{l}<<<X_{w}\right.}} s\left(z, x, z, \mathbf{X}_{-i k}\right) d^{n-2} \hat{F}\left(\mathbf{x}_{-i k} \mid z\right) d G(z) .
\end{aligned}
$$

In a similar fashion, we rewrite $\pi(x, y)$ : 


$$
\begin{aligned}
\pi(x, y)= & -\int_{\substack{s\left(z, y, z, \mathbf{x}_{-i k}\right)>0 \\
X_{l}<z<X_{w}}} s\left(z, y, z, \mathbf{x}_{-i k}\right) d G(z) d^{n-2} \hat{F}\left(\mathbf{x}_{-i k} \mid z\right)+ \\
& +\int_{\substack{s\left(x, y, z, \mathbf{X}_{-i k}\right)>0 \\
X_{l}<z<X_{w}}} s\left(x, y, z, \mathbf{x}_{-i k}\right) d G(z) d^{n-2} \hat{F}\left(\mathbf{x}_{-i k} \mid z\right) .
\end{aligned}
$$

Under our assumptions on functions $v$ and $F, \pi(x, y)$ is differentiable. By taking the partial derivative of $\pi(x, y)$ w.r.t. $y$, we have to consider variations of the integrands and of the domains of integration, i.e., variations of the sets of the values of $z$ and $\mathbf{X}_{-i k}$ where $s\left(z, y, z, \mathbf{x}_{-i k}\right)>0$ and $s\left(x, y, z, \mathbf{x}_{-i k}\right)>0$. Due to the continuity of $s\left(x, y, z, \mathbf{X}_{-i k}\right)$, all variations of domains happen at $s=0$ and do not contribute to $\pi_{y}(x, y)$. Thus,

$$
\begin{aligned}
\pi_{y}(x, y)= & -\int_{\substack{s\left(z, y, z, \mathbf{x}_{-i k}\right)>0 \\
X_{l}<z<X_{w}}} \frac{\partial}{\partial y} s\left(z, y, z, \mathbf{x}_{-i k}\right) d G(z) d^{n-2} \hat{F}\left(\mathbf{x}_{-i k} \mid z\right)+ \\
& +\int_{\substack{s\left(x, y, z, \mathbf{X}_{-i k}>0 \\
X_{l}<z<X_{w}\right.}} \frac{\partial}{\partial y} s\left(x, y, z, \mathbf{x}_{-i k}\right) d G(z) d^{n-2} \hat{F}\left(\mathbf{x}_{-i k} \mid z\right) .
\end{aligned}
$$

Next, using the definition of $s\left(x, y, z, \mathbf{X}_{-i k}\right)$ and writing $v_{k} \equiv \partial v / \partial X_{k}$ yields:

$$
\begin{aligned}
\pi_{y}(x, y)= & \int_{\substack{s\left(z, y, z, \mathbf{X}_{-i k}>0 \\
X_{l}<z<X_{w}\right.}} v_{k}\left(z,\left(y, \mathbf{x}_{-i k}\right)\right) d G(z) d^{n-2} \hat{F}\left(\mathbf{x}_{-i k} \mid z\right)- \\
& -\int_{\substack{s\left(x, y, z, \mathbf{X}_{-i k}\right)>0 \\
X_{l}<z<X_{w}}} v_{k}\left(z,\left(y, \mathbf{x}_{-i k}\right)\right) d G(z) d^{n-2} \hat{F}\left(\mathbf{x}_{-i k} \mid z\right) .
\end{aligned}
$$

Then, using the following chain of the equivalence relations:

$$
\begin{aligned}
s\left(z, y, z, \mathbf{X}_{-i k}\right)>0 & \Leftrightarrow v\left(z,\left(z, \mathbf{X}_{-i k}\right)\right)>v\left(z,\left(y, \mathbf{X}_{-i k}\right)\right) \Leftrightarrow z<y \\
& \Leftrightarrow v\left(y,\left(z, \mathbf{X}_{-i k}\right)\right)>v\left(z,\left(y, \mathbf{X}_{-i k}\right)\right),
\end{aligned}
$$

we rewrite $\pi_{y}(x, y)$ as follows

$$
\begin{aligned}
& \pi_{y}(x, y)=\int_{\substack{v\left(y,\left(z: \mathbf{X}_{-i k}\right)\right)>v\left(z,\left(y: \mathbf{X}_{-i k}\right)\right) \\
X_{1}<z<X_{w}}} v_{k}\left(z,\left(y, \mathbf{X}_{-i k}\right)\right) d G(z) d^{n-2} \hat{F}\left(\mathbf{x}_{-i k} \mid z\right)- \\
& -\int_{\substack{v\left(x,\left(z: \mathbf{X}_{-i k}\right)\right)>v\left(z,\left(y: \mathbf{X}_{-i k}\right)\right) \\
X_{l}<z<X_{w}}} v_{k}\left(z,\left(y, \mathbf{X}_{-i k}\right)\right) d G(z) d^{n-2} \hat{F}\left(\mathbf{x}_{-i k} \mid z\right) .
\end{aligned}
$$

Suppose now that $y>x$. This implies $v\left(y,\left(z, \mathbf{X}_{-i k}\right)\right)>v\left(x,\left(z, \mathbf{X}_{-i k}\right)\right)$ so that we can rewrite $\pi_{y}(x, y)$ as follows: 


$$
\begin{aligned}
& \pi_{y}(x, y)=\underbrace{}_{\substack{v\left(y,\left(z: \mathbf{X}_{-i k}\right)\right)>v\left(x,\left(z: \mathbf{X}_{-i k}\right)\right)>v\left(z,\left(y: \mathbf{X}_{-i k}\right)\right) \\
X_{1}<z<X_{w}}} v_{v}\left(z,\left(y, \mathbf{x}_{-i k}\right)\right) d G(z) d^{n-2} \hat{F}\left(\mathbf{x}_{-i k} \mid z\right)+ \\
& +\underbrace{}_{\substack{v\left(y,\left(z: \mathbf{X}_{-i k}\right)\right)>v\left(z,\left(y: \mathbf{X}_{-i k}\right)\right)>v\left(x,\left(z: \mathbf{X}_{-i k}\right)\right) \\
X_{l}<z<X_{w}}} v_{k}\left(z,\left(y, \mathbf{x}_{-i k}\right)\right) d G(z) d^{n-2} \hat{F}\left(\mathbf{x}_{-i k} \mid z\right)- \\
& -\int_{\substack{v\left(x,\left(z: \mathbf{X}_{-i k}\right)\right)>v\left(z,\left(y: \mathbf{X}_{-i k}\right)\right) \\
X_{l}<z<X_{w}}} v_{i-i k}\left(z,\left(y, \mathbf{x}_{-i}\right)\right) d G(z) d^{n-2} \hat{F}\left(\mathbf{x}_{-i k} \mid z\right) .
\end{aligned}
$$

The first and the last terms cancel each other out, so that

$$
\pi_{y}(x, y)=\int_{\substack{v\left(y,\left(z: \mathbf{X}_{-i k}\right)\right)>v\left(z,\left(y: X_{-i k}\right)\right)>v\left(x,\left(z: \mathbf{X}_{-i k}\right)\right) \\ X_{l}<z<X_{w}}} v_{k}\left(z,\left(y, \mathbf{x}_{-i k}\right)\right) d G(z) d^{n-2} \hat{F}\left(\mathbf{x}_{-i k} \mid z\right)<0 .
$$

The last inequality follows from $v_{k}<0$ and from the fact that the domain of integration is never empty. Indeed, for $z=y>x$ :

$$
v\left(y,\left(z, \mathbf{x}_{-i k}\right)\right)=v\left(z,\left(y, \mathbf{x}_{-i k}\right)\right)>v\left(x,\left(z, \mathbf{x}_{-i k}\right)\right),
$$

and for $z$ marginally lower than $y$ :

$$
v\left(y,\left(z, \mathbf{x}_{-i k}\right)\right)>v\left(z,\left(y, \mathbf{x}_{-i k}\right)\right) .
$$

Hence, $\pi_{y}(x, y)<0$ and, therefore, $\pi(x, y)<\pi(x, x)$ for all $y>x$. Thus, choosing $y>x$ is not a profitable deviation.

Similarly, $y<x$ implies $v\left(y,\left(z, \mathbf{X}_{-i k}\right)\right)<v\left(x,\left(z, \mathbf{X}_{-i k}\right)\right)$ so that we rewrite $\pi_{y}(x, y)$ as follows:

$$
\begin{aligned}
& \pi_{y}(x, y)=\int_{\substack{v\left(y,\left(z: \mathbf{X}_{-i k}\right) \\
X_{l}<z<X_{w}\right.}} \int_{>v\left(z,\left(y: \mathbf{X}_{-i k}\right)\right)} v_{k}\left(z,\left(y, \mathbf{x}_{-i k}\right)\right) d G(z) d^{n-2} \hat{F}\left(\mathbf{x}_{-i k} \mid z\right)- \\
& -\underset{\substack{v\left(x,\left(z: \mathbf{X}_{-i k}\right)\right)>v\left(y,\left(z: \mathbf{X}_{-i k}\right)\right)>v\left(z,\left(y: \mathbf{X}_{-i k}\right)\right) \\
X_{l}<z<X_{w}}}{ } \int_{k}\left(z,\left(y, \mathbf{x}_{-i k}\right)\right) d G(z) d^{n-2} \hat{F}\left(\mathbf{x}_{-i k} \mid z\right)- \\
& -\underbrace{}_{\substack{v\left(x,\left(z: \mathbf{X}_{-i k}\right)\right)>v\left(z,\left(y: \mathbf{X}_{-i k}\right)\right)>v\left(y,\left(z: \mathbf{X}_{-i k}\right)\right) \\
X_{l}<z<X_{w}}} v_{k}\left(z,\left(y, \mathbf{X}_{-i k}\right)\right) d G(z) d^{n-2} \hat{F}\left(\mathbf{x}_{-i k} \mid z\right),
\end{aligned}
$$

which yields:

$$
\pi_{y}(x, y)=-\underset{\substack{v\left(x,\left(z: \mathbf{X}_{-i k}\right)\right)>v\left(z,\left(y: X_{-i k}\right)\right)>v\left(y,\left(z: X_{-i k}\right)\right) \\ X_{l}<z<X_{w}}}{ } \int_{i} v_{k}\left(z,\left(y, \mathbf{x}_{-i k}\right)\right) d G(z) d^{n-2} \hat{F}\left(\mathbf{x}_{-i k} \mid z\right)>0 .
$$

Hence, neither $y<x$ is a profitable deviation.

On the other hand, setting fee $e_{i}$ above $e^{F E F}(1)$ is strictly suboptimal for the given off-the-equilibrium path beliefs as it affects neither the bid of the other bidders, nor the winning probability of bidder $i$, nor the auction price bidder $i$ pays if he wins. It only 
increases expenses and, therefore, is strictly suboptimal. Thus, no bidder has incentives to deviate from equilibrium fee $e^{F E F}(x)$.

\section{Proof of Proposition 2.}

Let bidder $i$ of type $X_{i}=x$ bid $\beta^{S P}(y)$ in the SP-auction, where $\beta^{S P}(x)$ is a monotonically increasing symmetric equilibrium bidding function. Then, bidder $i$ has expected value $\hat{v}(x, z, z)$ and wins if and only if he outbids the bid $\beta^{S P}(z)$ of bidder $k$ with type $X_{k}=z$. The expected profit of bidder $i$ is, therefore,

$$
\pi(x, y)=\int_{0}^{y}\left(\hat{v}(x, z, z)-\beta^{S P}(z)\right) d G(z) .
$$

Maximizing $\pi(x, y)$ w.r.t. $y$ yields the necessary first-order condition:

$$
\beta^{S P}(y)=\hat{v}(x, y, y),
$$

which must hold for $y=x$. Thus, if such an equilibrium does exist, it must be $\beta^{S P}(x)=\hat{v}(x, x, x)$.

Suppose now that all bidders follow $\beta^{S P}(x)=\hat{v}(x, x, x)$. Then

$$
\pi(x, y)=\int_{0}^{y}(\hat{v}(x, z, z)-\hat{v}(z, z, z)) d G(z) .
$$

It can be easily seen that $y=x$ is a global maximum of $\pi(x, y)$ w.r.t. $y$ because

$$
\pi_{y}(x, y)=(\hat{v}(x, y, y)-\hat{v}(y, y, y)) g(y)<0
$$

for $y>x$ and $\pi_{y}(x, y)>0$ for $y<x$. Hence, $\beta^{S P}(x)=\hat{v}(x, x, x)$ is a unique monotonically increasing symmetric equilibrium, provided $\hat{v}(x, x, x)$ monotonically increases in $x$.

The revenue raised in the SP-auction can be written as follows:

$$
\begin{aligned}
R^{S P} & =E\left(\beta^{S P}\left(X_{k}\right) \mid X_{i}>X_{k}, X_{w}>X_{k}, X_{l}<X_{k}\right)=N \int_{0}^{1}\left(\int_{0}^{x} \beta^{S P}(z) d G(z)\right) d F(x) \\
& =N \int_{0}^{1} \int_{0}^{x} \hat{v}(z, z, z) d G(z) d F(x) .
\end{aligned}
$$

In the FEF-auction, a part $R^{F E F, e}$ of the revenue comes from collecting the entry fees: 


$$
R^{F E F, e}=N \cdot E\left(e^{F E F}\left(X_{i}\right)\right)=N \int_{0}^{1} \int_{0}^{x}(\hat{v}(z, z, z)-\hat{v}(z, x, z)) d G(z) d F(x) .
$$

The remaining part $R^{F E F, \beta}$ of the revenue stems from the bids made:

$$
\begin{aligned}
R^{F E F, \beta} & =E\left(\beta^{F E F}\left(X_{k}, e_{k}, \mathbf{e}_{-k}\right) \mid X_{w}>X_{k}>X_{l}, X_{i}>X_{k}\right) \\
& =E\left(v\left(X_{k},\left(X_{i}, \mathbf{X}_{-i k}\right)\right) \mid X_{w}>X_{k}>X_{l}, X_{i}>X_{k}\right) \\
& =N \int_{0}^{1} \int_{0}^{x} E\left(v\left(X_{k},\left(X_{i}, \mathbf{X}_{-i k}\right)\right) \mid X_{w}>X_{k}=z>X_{l}, X_{i}=x>X_{k}\right) d G(z) d F(x) \\
& =N \int_{0}^{1} \int_{0}^{x} \hat{v}(z, x, z) d G(z) d F(x) .
\end{aligned}
$$

As $R^{F E F, e}+R^{F E F, \beta}=R^{S P}$, revenue equivalence holds.

\section{Proof of Proposition 3.}

First, it is easy to check that $e^{F E F}(x)$ strictly increases:

$$
\frac{d}{d x} e^{F E F}(x)=b F(x \mid x)>0 .
$$

This implies that $h(e)=\max \left\{x: e^{F E F}(x) \leq e\right\}$ is a proper inverse function, which leads to the Bayesian beliefs $B^{F E F}\left(x \mid e_{j}\right)$ on-the-equilibrium path.

Second, bidding one's own valuation is optimal given beliefs. In the rest of the proof, we show that bidders do not benefit by deviating from $e^{F E F}(x)$ for the proposed on- and off-the-equilibrium path beliefs.

If bidder $i$ of type $x$ sets entry fee $e^{F E F}(y)$, his expected profit $\pi(x, y)$ is

$$
\begin{aligned}
\pi(x, y) & \equiv-e^{F E F}(y)+\int_{0}^{(a x+b y) /(a+b)}(a x-b z-a z+b y) d F(z \mid x) \\
& =-b \int_{0}^{y} F(z \mid z) d z+(a+b) \int_{0}^{(a x+b y) /(a+b)} F(z \mid x) d z .
\end{aligned}
$$

Hence, if $\pi(x, y) \leq \pi(x, x)$ for all $y$ bidder $i$ has no incentives to deviate from $e^{F E F}(x)$. Differentiating $\pi(x, y)$ w.r.t. $y$ yields:

$$
\pi_{y}(x, y)=-b\left(F(y \mid y)-F\left(\frac{a x+b y}{a+b} \mid x\right)\right) .
$$


Under the assumption of the proposition, $\pi_{y}(x, y) \leq 0$ for $y>x$ and $\pi_{y}(x, y) \geq 0$ for $y \leq x$, so that $y=x$ is a global maximum. Hence, there are no profitable deviations from $e^{F E F}(x)$ below $e^{F E F}(1)$. For deviations above $e^{F E F}(1)$, the same argument as in the proof of Proposition 1 applies.

In order to derive the necessary condition for an equilibrium to exist we note that the global maximum $y=x$ of $\pi(x, y)$ w.r.t. $y$ must necessarily be a local maximum. The second-order condition for the local maximum is $\pi_{y, y}(x, x) \leq 0$ :

$$
\pi_{y, y}(x, x)=-b\left(\frac{d}{d x} F(x \mid x)-\frac{b}{a+b} f(x \mid x)\right) \leq 0,
$$

and the necessary condition of Proposition 3 follows.

\section{Proof of Proposition 4.}

Expected payment $M^{S P}(x)$ of bidder $i$ of type $x$ in the SP-auction is:

$$
\begin{aligned}
M^{S P}(x) & =G(x \mid x) E\left((a-b) X_{j}+c \mid X_{i}=x, X_{j} \leq x\right)=(a-b) \int_{0}^{x} z d G(z \mid x)+c G(x \mid x) \\
& =((a-b) x+c) G(x \mid x)-(a-b) \int_{0}^{x} G(z \mid x) d z .
\end{aligned}
$$

His expected payment in the FEF-auction is:

$$
\begin{aligned}
M^{F E F}(x) & =e^{F E F}(x)+G(x \mid x) E\left(a X_{j}-b X_{i}+c \mid X_{i}=x, X_{j}<x\right) \\
& =b \int_{0}^{x} G(z \mid z) d z+\int_{0}^{x}(a z-b x) d G(z \mid x)+c G(x \mid x) \\
& =((a-b) x+c) G(x \mid x)+b \int_{0}^{x} G(z \mid z) d z-a \int_{0}^{x} G(z \mid x) d z .
\end{aligned}
$$

The difference $M^{F E F}(x)-M^{S P}(x)$ is

$$
M^{F E F}(x)-M^{S P}(x)=b \int_{0}^{x}(G(z \mid z)-G(z \mid x)) d z>0
$$

for all $x>0$ because, due to affiliation, $G(z \mid z)>G(z \mid x)$ for all $z<x$. Therefore, the $e x-$ ante payment of any bidder, hence the auction revenue as well, is strictly higher in the FEF-auction than in the SP-auction. 